for their own jurisdictions and standards differ both within and between jurisdictions. Formerly in Europe, aeromedical standards were more prescriptive, now they are more risk based and open to interpretation. Pilots frequently change competent authority and jurisdiction; this presents a challenge to medical assessors. Due to recent adverse events, more aspects of pilot medical assessments are becoming mandatory including formal psychological assessment, drug and alcohol testing and peer support for both pilots and aeromedical examiners. There is also a drive to include health promotion and preventative medicine as an integral part of routine medical examinations for pilots. This has been strikingly absent to date as the routine medicals focus on a 'pass/fail' system. As with any change in aeromedicine, a way that is acceptable to all involved is being established currently. Regulatory aeromedicine is truly international largely due to the 'client' base and the many medical services and regulators they encounter around the world in the course of their careers. For these very reasons, it is also an exciting field to work in.

In March 2015 another 'unthinkable' happened in aviation with the Germanwings disaster. The world was shocked as events unfurled, and for commercial aviation the tragic situation had to be risk assessed with speed. Individual airlines considered their own risk assessment and the European Aviation Safety Agency, EASA, set up a Task Force in May 2015, their report being published in July 2015. The Task Force made the following 6 recommendations:

1. The principle of 'two persons in the cockpit at all time' should be maintained.

2. Pilots should undergo a psychological evaluation before entering airline service.

3. Airlines should run a random drugs and alcohol programme.

4. Robust programme for oversight of aeromedical examiners should be established.

5. A European aeromedical data repository should be created.

6. Pilot support systems should be implemented within airlines.

The French accident investigation report (BEA) was also published with its own recommendations. Such detailed analysis of all aircraft accidents is required to help improve flight safety and recommendations often follow, some more controversial than others. This presentation will review some of the recommendations in detail discussing the implications, implementation, and benefits to flight safety.

\section{HYPERBARIC MEDICINE (DEALING WITH EMPLOYEES WHO WORK UNDER PHYSICAL PRESSURE)}

AJ Bray*. SWIMS Ltd, Solihull, UK

\subsection{6/oemed-2018-ICOHabstracts. 1063}

Aim of special session Using 3 related employment scenarios to explore the increasing challenges to employees working under pressure and their medical attendants.

Presenters: M. Gonevski ${ }^{1}$, W. Robertson ${ }^{2}$, A. Heili ${ }^{3}$

${ }^{1}$ Midlands Diving Chamber, Rugby, UK

${ }^{2}$ Independent Occupational Physician, Yorkshire, England

${ }^{3}$ Hyperbaric Tunnelling and Medical Services- Romford, UK Description of content The scope of the working community who toil under increased physical pressure will be collectively addressed in this interactive workshop. New equipment, techniques and operating protocol will be debated to focus the delegates on scientific evidence and recent accident data.

The individual topics are inter-related and cover the breadth of the working practices of tunnelling, diving and hyperbaric chamber activities. The speakers will cover tunnelling technology, medical criteria for chamber staff and the threatening use of bizarre and dangerous diving breathing systems.

Following a brief historical analysis the individual presenters with provide updating material to set the scene and aid participation in constructive dialogue.

\section{0a QUALIFICATION AND REQUIREMENTS FOR HYPERBARIC CHAMBER FACILITY PERSONNEL}

M Gonevski. Midlands Diving Chamber, Rugby, UK

\subsection{6/oemed-2018-ICOHabstracts. 1064}

The range of personnel involved in hyperbaric chamber operations requires a specific set of skills. There are minimum requirements for all key level positions with regards to training, experience and medical fitness and these are discussed in detail. The appropriate staff numbers and their qualifications depend on the combination of patient numbers and needs, chamber type and features, as well as the use of medical and other equipment. For a standard multi-place chamber the minimum complement must comprise of a hyperbaric physician on-site, a minimum of one qualified attendant available inside and one outside the chamber, as well as a hyperbaric technical officer. Depending on the type of patients being treated, there may be a necessity for an intensive care trained nurse as hyperbaric attendant. The qualifications and responsibilities can overlap between the hyperbaric facility personnel. The training requirements are also very specific. The Hyperbaric Physician must have knowledge, training and experience in the diagnosis, treatment and assessment of individuals in whom hyperbaric oxygen therapy is contemplated. He is medically accountable for the safety of patients and staff involved in the treatment. This requires knowledge of the indications, contraindications, side effects and complications of therapy, as well as provisions of an environment to safely treat patients. The Hyperbaric Attendant must be a nurse or a diving medical technician with emergency medical training. They must possess both theoretical and practical knowledge and competencies. This is achieved through successful completion of a training course that covers specific relevant curriculum. Before any medical personnel can enter hyperbaric environment they must be certified as medically fit to undergo pressurisation. The person's fitness is determined through a medical examination that comprises of a detailed medical questionnaire and a medical examination conducted by a medical practitioner appropriately trained in hyperbaric and diving medicine.

\section{0b HOOKA DIVING SYSTEMS}

W Robertson. Independent Occupational Physician, Yorkshire, UK

10.1136/oemed-2018-ICOHabstracts. 1065

Diving is an incredibly common activity both in the recreational and commercial domain. Despite being an activity 